\title{
A New Definition of Conformal and Projective Infinity of Space-Times
}

\author{
B. G. Schmidt \\ Max-Planck-Institut für Physik und Astrophysik, \\ München, Federal Republic of Germany \\ Received October 10, 1973; in revised form January 7, 1974
}

\begin{abstract}
Any conformal or projective structure on a manifold $\mathscr{M}$ defines a natural boundary $\partial \mathscr{M}$. For Minkowski space these coincide with null infinity as defined by Penrose and projective infinity as defined by Eardley and Sachs, respectively.
\end{abstract}

\section{§ 1. Introduction}

The $b$-boundary construction $[1,2]$ is a device to attach to any spacetime $\mathscr{V}^{4}$ a boundary $\partial_{b} \mathscr{V}^{4}$, which is intrinsically defined by the Lorentz metric of $\mathscr{V}^{4}$. In this paper it will be shown that a similar construction is possible for a conformal or projective structure on a manifold. Thus one gets for any space time two boundaries $\partial_{c} \mathscr{V}^{4}, \partial_{p} \mathscr{V}^{4}$ which are determined by the conformal and projective structures of $\mathscr{V}^{4}$.

It turns out that the conformal boundary of Minkowski space determined by this method is $\mathscr{I}$ as defined by Penrose [3], together with the points $I^{-}, I^{\circ}, I^{+}$. In the projective case one finds that the boundary of Minkowski space is the same as projective infinity defined recently by Eardly and Sachs $[4,5]$. Therefore we get as well a new definition of $\mathscr{I}$ and future projective infinity, as at the same time a genuine generalisation, because the boundaries are defined for any space.

Therefore it is within this framework a well defined question for example whether the null generators of $\mathscr{I}^{+}$of an asymptotically simple space-time $\mathscr{V}^{4}$ have a past end point in $\partial_{c} \mathscr{V}^{4}$ or not.

Hopefully this method will lead to some new insight into the structure of asymptotically simple spaces near $I^{0}$ and $I^{+}$.

The mathematical background - which will not be used in this paper is the theory of prolongations of $G$-structures. If one considers all metrics compatible with a given conformal structure, then their connections define a reduction of the frame bundle of the frame bundle. It turns out that on this bundle there exists a parallelisation, determined intrinsically by the conformal structure. Hence one gets a positive definite metric, 
forms the Cauchy completion to obtain a boundary of the bundle, and projects this boundary down to get the boundary of the base space. The interpretation of boundary points in terms of the space-time is more complicated than in the case of the $b$-boundary. Roughly speaking it is the following: A curve $x(\lambda), 0 \leqq \lambda<1$ which is inextensible in $\mathscr{V}^{4}$ terminates at a point of $\partial_{c} \mathscr{V}^{4}$ if a metric $g$ exists in the conformal class such that 1) the Ricci tensor of $g$ vanishes on $x(\lambda)$ for $\lambda_{0} \leqq \lambda<1,2$ ) the generalised affine length [2] of $x(\lambda), \lambda_{0} \leqq \lambda<1$, calculated with the connection of $g$ is finite.

The plan of the paper is the following: In Section 2 the definition of the conformal boundary is developed. Section 3 contains the proof that for Minkowski space $\partial_{c} \mathscr{V}^{4}=\mathscr{I} \cup I^{-} \cup I^{0} \cup I^{+}$. The interpretation of boundary points is given in Section 4 . Finally there are some remarks on the projective case in Section 5. Appendix 3 contains a geometrical proof that the conformal group of motions of the Einstein Universe is a covering group of $\mathcal{O}(4,2)$.

\section{§ 2. Definition of the Conformal Boundary of a Space-Time}

In this section it will be shown that a conformal structure on a manifold $\mathscr{M}$ defines in a natural way two principal bundles over $\mathscr{M}$ and a parallelisation on one of these, which then will be used to define a boundary of $\mathscr{M}$ in perfect analogy to the $b$-boundary [1]. The general mathematical background is the theory of prolongations of $G$-structures as developed in $[6,7]$. The essential geometrical ideas, however, were already known to Cartan like nearly anything in local Differential Geometry.

In the treatment given here, the general theory of prolongations will not be used in the hope that a more direct treatment will be better understandable to Relativists.

Let $\mathscr{M}^{4}$ be a manifold. A conformal structure $\mathscr{C}$ on $\mathscr{M}^{4}$ is defined as an equivalence class of Lorentz metrics defined by the relation $g \sim \bar{g}$ if $\bar{g}=e^{2 \sigma} g$, where $\sigma$ is any real-valued function on $\mathscr{M}$. Let us write $g \in \mathscr{C}$ for a metric in the conformal class. A Lorentz metric defines a reduction of the frame bundle $\mathscr{L}(\mathscr{M})$ to the bundle of orthonormal frames. In the same way, a conformal structure $\mathscr{C}$ gives a reduction of $\mathscr{L}(\mathscr{M})$ defined by all frames which are orthonormal for some metric $g \in \mathscr{C}$. This bundle is denoted by $\mathscr{P}(\mathscr{M})^{1}$ and its structure group is

$$
\mathscr{C} \mathcal{O}:=\left\{a \mid a \in \mathscr{G} l(4, \mathscr{R}), \eta(a \xi, a \bar{\xi})=c^{2} \eta(\xi, \bar{\xi})\right\}
$$

\footnotetext{
${ }^{1}$ One can define $\mathscr{C}$ as a reduction of $\mathscr{L}(\mathscr{M})$ with structure group $\mathscr{C} \mathscr{O}$.
} 
when $\eta$ is a metric of Lorentz signature on the vector space $\mathscr{R}^{4}$. Clearly $\mathscr{C} \mathcal{O}$ is the direct product of the Lorentz group and $\mathscr{R}^{+}$, the group of positive real numbers under multiplication.

Hence $u \in \mathscr{P}(\mathscr{M})$ is a frame $\left(e_{a}\right)$ at a point $x \in \mathscr{M}$ which is orthonormal relative to some $g \in \mathscr{C}$. Any other frame at $x$ which is in $\mathscr{P}(\mathscr{M})$ is then given by $\bar{e}_{b}=e_{a} a_{b}^{a}$ with $a_{a}^{b} \in \mathscr{C} \mathcal{O}$.

In the following $\mathscr{P}(\mathscr{M})$ will always be considered as a subset of $\mathscr{L}(\mathscr{M})$.

Choose $g \in \mathscr{C}$. Then $g$ determines uniquely a torsion free connection $\Gamma$ on $\mathscr{L}(\mathscr{M})$. Denote by $\left\{H_{u}\right\}$ the collection of horizontal subspaces defining $\Gamma$, and by $B_{i}$ the standard horizontal fields, $[8,1]$, which are defined by

$$
\left(B_{i}\right)_{u} \in H_{u}, \pi_{*}\left(B_{i}\right)_{u}=e_{i} \text { if } u=\left(e_{i}\right)
$$

A metric $g$ determines uniquely a horizontal subspace at any point of $\mathscr{L}(\mathscr{M})$. In contrast to this we get from a conformal structure a whole collection of horizontal subspaces at any point.

What is the relation between $H_{u}, H_{u}^{\prime}$ ? If $H_{u}$ is defined by $g \in \mathscr{C}, H_{u}^{\prime}$ by $e^{2 \sigma} g$, then the relation between the Christoffel symbols in a local coordinate system is [9]

$$
\Gamma_{i k}^{\prime l}=\Gamma_{i k}^{l}+\delta_{i}^{l} \sigma_{\mid k}+\delta_{k}^{l} \sigma_{\mid i}-g_{i k} g^{l s} \sigma_{\mid s} .
$$

From this and the expression for $B_{i}$ in coordinates $[2,10]$ one finds immediately

$$
B_{i}^{\prime}=B_{i}-\left(\delta_{i}^{l} \tilde{\sigma}_{\mid k}+\delta_{k}^{l} \tilde{\sigma}_{\mid i}-\tilde{g}_{i k} \tilde{g}^{l s} \tilde{\sigma}_{\mid s}\right) E_{l}^{*}
$$

Here $\stackrel{*}{E}_{k}^{l}$ denote the standard vertical vector fields in $\mathscr{L}(\mathscr{M})$ and $\tilde{\sigma}_{i k}, \tilde{g}_{k l}$ are functions defined on $\mathscr{L}(\mathscr{M})$ in the following way: if $g(X, Y)$ is a tensor field on $\mathscr{M}$, then $\tilde{g}_{i k}(u)$ is defined by $\tilde{g}_{i k}(u)=g\left(X_{i}, X_{k}\right)$ if $u=\left(X_{i}\right)$. Hence $\tilde{\sigma}_{\mid k}(u), \tilde{g}_{i k}(u)$ are just the components of $d \sigma$ and $g$ in the frame $u$. (This convention will be used for any tensor field on $\mathscr{M}$.) In particular $\tilde{g}_{i k} \tilde{g}^{l s}$ is constant on $\mathscr{P}(\mathscr{M})$, i.e. for conformal frames; more precisely $\tilde{g}_{i k} \tilde{g}^{l s}$ $=\eta_{i k} \eta^{l s}$ where $\eta_{i k}$ is diagonal $(-1,1,1,1)$ as well as $\eta^{i k}$. Therefore we find that at $u_{0} \in \mathscr{P}(\mathscr{M})$ the preferred class of horizontal subspaces is spanned by

$$
\left(B_{i}^{\prime}\right)_{u_{0}}=\left(B_{i}\right)_{u_{0}}-\left(\delta_{i}^{l} b_{k}+\delta_{k}^{l} b_{i}-\eta_{i k} \eta^{l s} b_{s}\right) E_{l}^{*} .
$$

The vertical fields $E_{\alpha}^{*}, \alpha=1, \ldots, 7$ in $\mathscr{P}(\mathscr{M})$ are given by $(k<l)$

$$
\stackrel{*}{*}_{l}^{k}=\stackrel{*}{E}_{l}^{k}-\eta_{l t} \eta^{k s} \stackrel{*}{t}_{s}^{t}, \quad \delta_{k}^{i} \stackrel{*}{E}_{i}^{k}=\stackrel{*}{E}_{k}^{k}
$$

since the Lie algebra of $\mathscr{C} \mathcal{O}$ is spanned by the Lie algebra $E_{l}^{k}-\eta_{l t} \eta^{k s} E_{s}^{t}$ of the Lorentz group and $\delta_{k}^{i}\left(E_{l}^{k}\right.$ is the matrix with 1 in the $k$-th row and $l$-th column and 0 at all other entries). 
Now we realise that a conformal structure defines a collection of frames of $\mathscr{P}(\mathscr{M})$. At $u_{0} \in \mathscr{P}(\mathscr{M})$ these frames are given by (2.5), (2.6) with $b_{k} \in \mathscr{R}^{4}$.

With the abbreviation

$$
S_{i k}^{l}(b):=\delta_{i}^{l} b_{k}+\delta_{k}^{l} b_{i}-\eta_{i k} \eta^{l s} b_{s}
$$

any two of the preferred frames at $u_{0}$ are related by a linear transformation

$$
\begin{aligned}
& B_{i}^{\prime} \rightarrow B_{i}^{\prime}-S_{i k}^{l}(b) E_{l}^{k}, \\
& E_{\alpha}^{*} \rightarrow E_{\alpha}^{*} .
\end{aligned}
$$

These transformations form an abelian vector group isomorphic to $\mathscr{R}^{4}$.

We now come to our $2^{\text {nd }}$ bundle. Considering the frame bundle of $\mathscr{P}(\mathscr{M})$ we realise that a conformal structure defines, via the preferred subspaces on $\mathscr{P}(\mathscr{M})$, a reduction of $\mathscr{L}(\mathscr{P}(\mathscr{M}))$ which we denote by $\mathscr{P}^{1}(\mathscr{P})$. The structure group of $\mathscr{P}^{1}(\mathscr{P})$ is $\mathscr{R}^{4}$. [The fact that the frames (2.5), (2.6) really form a reduction of $\mathscr{L}(\mathscr{P})$ follows easily because a connection $\Gamma$ of a metric $g \in \mathscr{C}$ defines a cross section $\lambda: \mathscr{P}(\mathscr{M}) \rightarrow \mathscr{L}(\mathscr{P}(\mathscr{M}))$.] Denote by $\pi^{1}$ the projection $\pi^{1}: \mathscr{P}^{1}(\mathscr{P}) \rightarrow \mathscr{P}(\mathscr{M})$.

Let us recapitulate the interpretation of a point $z_{0} \in \mathscr{P}^{1}$. It describes a point $u_{0}=\pi^{1}\left(z_{0}\right) \in \mathscr{P}(\mathscr{M})$ and a horizontal subspace $H_{u_{0}}$ which belongs to the distribution of horizontal subspaces of a connection of a metric $g \in \mathscr{C}$.

Another way of saying the same thing is this: $z_{0} \in \mathscr{P}^{1}$ determines uniquely (and is determined by) an equivalence class of metrics in $\mathscr{C}$ which satisfy $g^{\prime}=e^{2 \sigma} g$ and $\sigma\left(x_{0}\right)=0,(d \sigma)\left(x_{0}\right)=0$, where $x_{0}=\left(\pi \circ \pi^{1}\right)\left(z_{0}\right)$.

Or still another version: $z_{0} \in \mathscr{P}^{1}$ determines a frame $u_{0}=\pi^{1}\left(z_{0}\right)$ at $x_{0}=\pi\left(u_{0}\right)$ and a connection at $x_{0}$.

The essential point is that there exists a parallelisation on $\mathscr{P P}^{1}(\mathscr{P})$ which is uniquely determined by the conformal structure on $\mathscr{M}$.

We just repeat in a certain sense what we did before. $\mathscr{P}^{1}(\mathscr{P})$ is a principle bundle over $\mathscr{P}$ and we ask whether we can find preferred complements to the fibres.

Pick an arbitrary point $z_{0} \in \mathscr{P}^{1}$ and consider all $g \in \mathscr{C}$ such that the cross sections $\mathscr{P} \rightarrow \mathscr{P}^{1}$ defined by their connections pass through $z_{0}$. These cross sections are given by the fields

$$
\left(B_{i}^{\prime}\right)_{u}=\left(B_{i}\right)_{u}-\left(\delta_{i}^{l} \tilde{\sigma}_{\mid k}+\delta_{k}^{l} \tilde{\sigma}_{\mid i}-\eta_{i k} \eta^{l s} \tilde{\sigma}_{\mid s}\right) \stackrel{*}{E}_{l}^{k},
$$

hence are determined by $u \rightarrow \tilde{\sigma}_{\mid k}(u)$ and the fields $\stackrel{*}{\alpha}_{\alpha}$. The condition that all sections pass through the same point $z_{0}$ is given by $\tilde{\sigma}_{\mid k}\left(u_{0}\right)=b_{k}$ if $u_{0}=\pi^{1}\left(z_{0}\right)$. We can without loss of generality assume that $b_{k}=0$. Two 
such sections determine the same complement $H_{z_{0}}$ to the fibres in $\mathscr{P}^{1}$ if and only if

$$
\left(B_{i}\right)_{u_{0}} \tilde{\sigma}_{\mid k}=\left(B_{i}\right)_{u_{0}} \tilde{\sigma}_{\mid k}^{\prime}, \quad \stackrel{*}{E}_{\alpha} \tilde{\sigma}_{\mid k}=\stackrel{*}{E}_{\alpha} \tilde{\sigma}_{\mid k}^{\prime}
$$

holds. Now $\tilde{\sigma}_{\mid k}\left(u_{0}\right)=0$ implies $\left({ }^{*} \tilde{\sigma}_{\mid k}\right)\left(u_{0}\right)=0$, and by the very definition of the covariant derivative it follows that

$$
\left.\left(B_{i}\right)_{u_{0}} \tilde{\sigma}_{\mid k}=\widetilde{\left(\sigma_{\mid k \| i}\right.}\right)\left(u_{0}\right)
$$

where the covariant derivative is performed with the connection determined by $\left(B_{i}\right)_{u_{0}}$.

Therefore we find that there is a $1-1$ relation between $\widetilde{\sigma_{|i| \mid k}}\left(u_{0}\right)$ and a certain class of horizontal subspaces $H_{z_{0}}$.

Take now one of the sections $u \rightarrow \tilde{\sigma}_{\mid k}(u)$ and the corresponding fields $B_{i}^{\prime}$. Then

$$
\left[B_{i}^{\prime}, B_{k}^{\prime}\right]=-\tilde{R}_{t i k}^{s} E_{s}^{t}
$$

where $\tilde{R}_{t i k}^{s}$ are the components of the Riemann tensor in the frame $u$ of the metric defining the connection given by $B_{i}^{\prime}$.

Clearly $\left[B_{i}^{\prime}, B_{k}^{\prime}\right]_{u_{0}}$ depends only on $H_{z_{0}}$, the tangent space of the section $\tilde{\sigma}_{\mid k}(u)$. Can we find a unique $H_{z_{0}}$ by imposing conditions on $\tilde{R}_{t i k}^{s}$ ?

The Riemann tensor $R_{t i k}^{s}$ of a Lorentz metric decomposes uniquely into the conformal tensor $C_{t i k}^{s}$ and terms determined by the Ricci tensor $R_{i k}[11]$. More precisely

$$
R_{t i k}^{s}=C_{t i k}^{s}+S_{t i k}^{s}
$$

where $S_{t i k}^{s}$ is uniquely determined by the Ricci tensor and $C_{t i k}^{s}$ is the same for all metrics $e^{2 \sigma} g$.

Hence the brackets $\left[B_{i}^{\prime}, B_{k}^{\prime}\right]_{u_{0}}$ will all define the same conformal tensor, but different Ricci tensors depending on $\widetilde{\sigma_{\mid i \| k}}\left(u_{0}\right)$.

A direct calculation (or consultation of $[9,11]$ ) gives the following relation between the Ricci tensors of $\bar{g}=e^{2 \sigma} g$ and $g(n=4)$

$$
\bar{R}_{i k}=R_{i k}+2 \sigma_{|i| \mid k}-2 \sigma_{\mid i} \sigma_{\mid k}+g_{i k} g^{r s}\left(\sigma_{|r| \mid s}+2 \sigma_{\mid r} \sigma_{\mid s}\right),
$$

or, if we consider the corresponding functions on $\mathscr{P}$

$$
\tilde{\bar{R}}_{i k}=\tilde{R}_{i k}+2 \widetilde{\sigma_{|i| \mid k}}-2 \tilde{\sigma}_{\mid i} \tilde{\sigma}_{\mid k}+\tilde{g}_{i k} \tilde{g}^{r s}\left(\tilde{\sigma}_{|r| \mid s}+2 \tilde{\sigma}_{\mid r} \tilde{\sigma}_{\mid s}\right) .
$$

Hence there exists always $\tilde{\sigma}_{\mid r \| s}\left(u_{0}\right)$ such that $\tilde{\bar{R}}_{i k}\left(u_{0}\right)=0$.

This relation now implies that there exists a unique $H_{z_{0}}$ such that

$$
\left[B_{i}^{\prime}, B_{k}^{\prime}\right]_{u_{0}}=-C_{r i k}^{s}\left(u_{0}\right) \stackrel{E_{s}^{r}}{ } \text {. }
$$

Hence we find a uniquely determined distribution of horizontal subspaces $H_{z}$ on $\mathscr{P}^{1}(\mathscr{P})$. 
Let us reformulate the way in which $H_{z_{0}}$ is fixed in terms of $\mathscr{M}$ and metrics of the conformal class: Let $x_{0} \in \mathscr{M}$ and $g \in \mathscr{C}$ be arbitrary. Then there exist a conformal factor $e^{2 \sigma}$ such that $\sigma\left(x_{0}\right)=0, \sigma_{\mid k}\left(x_{0}\right)=0$, $\bar{R}_{i k}\left(x_{0}\right)=0$ where $\bar{R}_{i k}$ is the Ricci tensor of $e^{2 \sigma} g$; any two such conformal factors $\sigma, \sigma^{\prime}$ satisfy $\sigma_{|i| k}\left(x_{0}\right)=\sigma_{|i| k}^{\prime}\left(x_{0}\right)$ (in any coordinate system).

From the distribution of horizontal subspaces one immediately obtains a parallelisation:

Choose a basis $A^{m} \in \mathscr{R}^{4}$ and denote by $\stackrel{*}{A}^{m}$ the corresponding vector fields tangent to the fibres in $\mathscr{P}^{1}(\mathscr{P})$.

For any $z \in \mathscr{P}^{1}$ we define vector fields $Z_{i}$ and $Z_{\alpha}$ by the condition:

and

$$
\left(Z_{i}\right)_{z},\left(Z_{\alpha}\right)_{z} \in H_{z}
$$

$$
\begin{aligned}
& \pi_{*}^{1} Z_{i}=\left(B_{i}\right)_{u} \quad \text { if } \quad z=\left(B_{i}, \stackrel{E}{\alpha}_{\alpha}\right)_{u}, \\
& \pi_{*}^{1} Z_{\alpha}=\left(E_{\alpha}^{*}\right)_{u} \text { if } z=\left(B_{i}, E_{\alpha}^{*}\right)_{u} \text {. }
\end{aligned}
$$

Clearly $\stackrel{*}{ }^{m}, Z_{i}, Z_{\alpha}$ define a parallelisation on $\mathscr{P}^{1}$ which can be used to define a positive definite metric on $\mathscr{P}^{1}$ which is determined by the conformal structure $\mathscr{C}$ on $\mathscr{M}$.

The Cauchy completion of $\mathscr{P}^{1}$ gives the boundary points for $\mathscr{P}^{1}$, and the problem which remains to be solved is how to project the boundary down to $\mathscr{M}$. This can be achieved in the following way.

$\mathscr{P}^{1}(\mathscr{P})$ was defined as a bundle over $\mathscr{P}$ with projection $\pi^{1}: \mathscr{P}^{1} \rightarrow \mathscr{P}$. $\mathscr{P}$ is a bundle over $\mathscr{M}$ with projection $\pi: \mathscr{P} \rightarrow \mathscr{M}$. We can however also consider $\mathscr{P}^{1}$ as a bundle over $\mathscr{M}$ with projection $\pi \circ \pi^{1}: \mathscr{P}^{1} \rightarrow \mathscr{M}$, and it turns out that $\mathscr{P}^{1}$ is in fact a principle bundle over $\mathscr{M}$. To show this we have to define a group action on $\mathscr{P}^{1}$ such that the orbits are $\left(\pi \circ \pi^{1}\right)^{-1}(x)$ for $x \in \mathscr{M}$. The conformal group acts on $\mathscr{P}(\mathscr{M})$ on the right. Let $R_{a}: \mathscr{P}(\mathscr{M})$ $\rightarrow \mathscr{P}(\mathscr{M})$ be such a map for $a \in \mathscr{C} \mathcal{O}$. Then $R_{a}$ induces a transformation $\mathscr{P}^{1} \rightarrow \mathscr{P}^{1}$ by the differential of $R_{a}:$

$$
\left(R_{a}\right)_{*}: T_{u}(\mathscr{P}) \rightarrow T_{R_{a}(u)}(\mathscr{P}) .
$$

Let $H_{u}$ be a point of $\mathscr{P}^{1}$; then the horizontal subspace $\left(R_{a}\right)_{*} H_{u}$ defines again a point of $\mathscr{P}^{1}$, because there exists a metric connection $\Gamma$ on $P$ such that $H_{u}$ is a horizontal subspace of $\Gamma$ and therefore $\left(R_{a}\right)_{*} H_{u}=H_{u_{a}} \in \Gamma$, which implies that it is in $\mathscr{P}^{1}$. Therefore we have an action of $\mathscr{C} \mathscr{O}$ on $\mathscr{P}^{1}$ which is free. In Appendix 1 it will be proved that this action of $\mathscr{C} O$ and the action of $\mathscr{R}^{4}$ make $\mathscr{P}^{1}$ into a principle bundle over $\mathscr{M}$. It will furthermore turn out that $\stackrel{*}{A}^{m}, Z_{\alpha}$ are the fundamental vertical fields.

Denote by $\mathscr{G}$ the structure group of $\mathscr{P}^{1}(\mathscr{M})$. Then it will be proved in Appendix 2 that $R_{g}: \mathscr{P}^{1} \rightarrow \mathscr{P}^{1}$ is uniformly continuous with respect to the distance $d\left(z, z^{\prime}\right)$ defined by the positive definite metric on $\mathscr{P}^{1}$. 
Therefore the action $R_{g}: \mathscr{P}^{1} \rightarrow \mathscr{P}^{1}$ can be uniquely extended to the Cauchy completion $\bar{R}_{g}: \overline{\mathscr{P}}^{g} \rightarrow \overline{\mathscr{P}}^{1}$ [12].

We define $\overline{\mathscr{M}}:=\mathscr{M} \cup \partial_{c} \mathscr{M}$ as the quotient space $\overline{\mathscr{P}}^{1} / \mathscr{G}=: \overline{\mathscr{M}}$ with the usual topology.

In the next section it will be shown that $\partial_{c} \mathscr{M} \neq \emptyset$ for the conformal structure defined by Minkowski space and, it turns out that $\partial_{c} \mathscr{M}=\mathscr{I}$ $\cup I^{+} \cup I^{-} \cup I^{0}$.

In [15] a construction is given which assigns to any space-time a collection of boundary points which depends only on the causal structure. This boundary is in general different from the one constructed above because the boundary is not empty for the causal structure of the Einstein universe, whereas the conformal bundle boundary of the Einstein universe is empty (Lemma $3, \S 3$ ).

\section{§ 3. The Conformal Bundle Boundary of Minkowski Space}

Consider the Einstein universe $\mathscr{E}^{4}$, a space-time whose metric is

$$
-d t^{2}+d \Sigma^{2}(3), \quad t \in \mathscr{R}
$$

where $d \Sigma^{2}(3)$ is the metric of a unit 3-sphere. As shown by Penrose in several places [3,13], there exists an open submanifold $\mathscr{M}^{4}$ of $\mathscr{E}^{4}$ which is conformally isometric to Minkowski space. $\mathscr{M}^{4}$ can be described in the following way: Choose a point $I^{0} \in \mathscr{E}^{4}$ arbitrarily. Then the future light cone $\mathscr{I}^{+}$and the past light cone $\mathscr{I}^{-}$of $I^{0}$ refocus again at points $I^{+}, I^{-}$. $\mathscr{M}^{4}$ is the set of points in $\mathscr{E}^{4}$ which can be joined to $I^{0}$ without intersecting $\mathscr{I}^{+} \cup \mathscr{I}^{-} \cup I^{+} \cup I^{-}$and starting at $I^{0}$ in a spacelike direction.

Applying the results of $\S 2$, the bundle $\mathscr{P}^{1}\left(\mathscr{E}^{4}\right)$ together with a positive definite metric exists over $\mathscr{E}^{4}$, and the open subbundle $\left(\pi \circ \pi^{1}\right)^{-1}\left(\mathscr{M}^{4}\right)$ is clearly isometric to $\mathscr{P}^{1}\left(\mathscr{M}^{4}\right)$, the bundle over Minkowski space. We will now prove the following

Theorem. The conformal boundary of Minkowski space is

$$
\mathscr{I}^{+} \cup \mathscr{I}^{-} \cup I^{+} \cup I^{-} \cup I^{0} .
$$

To show this we need several Lemmas.

Lemma 1. If $\Phi: \mathscr{M} \rightarrow \mathscr{M}$ is a conformal isometry, then $\Phi$ induces a diffeomorphism $\tilde{\Phi}^{1}: \mathscr{P}^{1} \rightarrow \mathscr{P}^{1}$ leaving the parallelisation invariant. If $\tilde{\Phi}^{1}\left(z_{0}\right)=z_{0}$ then $\Phi$ is the identity on $\mathscr{M}$.

Proof. Any diffeomorphism $\Phi: \mathscr{M} \rightarrow \mathscr{M}$ induces a diffeomorphism $\tilde{\Phi}: \mathscr{L}(\mathscr{M}) \rightarrow \mathscr{L}(\mathscr{M})$, defined in the following way: if $u$ is the frame $\left(e_{a}\right)$ at $x$ then $\tilde{\Phi}(u)$ is the frame $\left(\Phi_{*} e_{a}\right)$ at $\Phi(x)$. If $\Phi$ is a conformal isometry, $\widetilde{\Phi}$ maps $\mathscr{P}(\mathscr{M})$, the bundle of conformal frames, into itself. 
Now $\tilde{\Phi}$, being a diffeomorphism $\mathscr{P} \rightarrow \mathscr{P}$, induces again a diffeomorphism $\tilde{\Phi}^{1}: \mathscr{L}(\mathscr{P}) \rightarrow \mathscr{L}(\mathscr{P})$.

Let $\Gamma$ be the connection of any $g \in \mathscr{C}$ and $H_{u}$ its distribution of horizontal subspaces. Then $\tilde{\Phi}_{*} H_{u}=: H_{\tilde{\Phi}(u)}$ is the distribution of horizontal subspaces of the connection of the metric $\Phi^{*} g=e^{2 \sigma} g$, as $\Phi$ is a conformal isometry. Hence $H_{\tilde{\Phi}(u)}^{\prime}$ is a horizontal subspace of the preferred class in $\mathscr{P}(\mathscr{M})$ and this implies that $\tilde{\Phi}_{1}$ maps $\mathscr{P}^{1}(\mathscr{P})$ onto itself.

Finally we have to show that $\tilde{\Phi}^{1}$ leaves the parallelisation defined on $\mathscr{P}^{1}$ invariant. Let $z_{0}$ be any point in $\mathscr{P}^{1}$ and $g \in \mathscr{C}$ a metric with vanishing Ricci tensor Ric at $x_{0}:=\left(\pi \circ \pi^{1}\right)\left(z_{0}\right)$. Then $\Gamma$, the connection defined by $g$, defines a cross-section in $\mathscr{P}^{1}(\mathscr{P})$ which is tangent to $H_{z_{0}}$, the preferred subspace. Let $\overline{\mathrm{Ric}}$ be the Ricci tensor of $\Phi^{*} g$; then $\overline{\mathrm{Ric}}$ $=\Phi^{*}$ Ric and therefore $\operatorname{Ric}\left(x_{0}\right)=0$ implies $\overline{\operatorname{Ric}}\left(\Phi\left(x_{0}\right)\right)=0$. Hence the connection of $\Phi^{*} g$ defines a section in $\mathscr{P}^{1}$ which is tangent to the preferred $H_{\tilde{\Phi}^{1}\left(z_{0}\right)}$. Because the section of $\Phi^{*} g$ is the image of the section of $g$, this implies that $\tilde{\Phi}^{1}$ leaves the preferred subspace $H_{z}$ invariant.

From this we get immediately that $\tilde{\Phi}_{*}^{1} Z_{i}=Z_{i}, \tilde{\Phi}_{*}^{1} Z_{\alpha}=Z_{\alpha}$. Finally $\tilde{\Phi}_{*}^{1} A^{m}=A^{m}$ follows because $\tilde{\Phi}^{1}$ commutes with the action of $\mathscr{R}^{4}$ on the fibres in $\mathscr{P}^{1}$.

As $\tilde{\Phi}^{1}$ leaves the parallelisation on $\mathscr{P}^{1}$ invariant it follows that $\tilde{\Phi}^{1}\left(z_{0}\right)=z_{0} \Rightarrow \tilde{\Phi}^{1}=\mathrm{id}$, and therefore using the definition of $\tilde{\Phi}^{1}$ we get $\Phi=\mathrm{id}$.

Remark. Lemma 1 implies in particular that the dimension of the group of conformal isometries of a space-time is at most 15 because $\operatorname{dim} \mathscr{P}^{1}\left(\mathscr{M}^{4}\right)=15$.

Next we need

Lemma 2. The conformal structure of the Einstein universe is invariant under a 15-parameter group of conformal motions acting transitively.

This is proved in [14]. A more geometrical proof is given in Appendix 3 .

This lemma shows that the conformal structure of the Einstein universe is for conformal structures what Minkowski space is for Lorentz metrics; it is conformally flat, has maximal conformal symmetry, and is simply connected.

Lemma 3. The conformal boundary of the Einstein universe is empty.

Proof. This is now an immediate consequence of Lemma 1, 2. The space $\mathscr{P}^{1}\left(\mathscr{E}^{4}\right)$ together with the metric defined by the parallelisation is a positive definite homogeneous space, hence complete.

Let us now prove the theorem. Lemma 3 implies that any curve of finite length without endpoint in $\mathscr{P}^{1}\left(\mathscr{M}^{4}\right) \subset \mathscr{P}^{1}\left(\mathscr{E}^{4}\right)$ projects on a curve in $\mathscr{E}^{4}$ which has an endpoint on $\partial_{\mathscr{E}} \mathscr{M}^{4}$, the boundary of $\mathscr{M}^{4}$ relative to 
$\mathscr{E}^{4}$. Clearly two curves in $\mathscr{M}^{4}$ defining the same boundary point in $\partial_{c} \mathscr{M}^{4}$, the conformal boundary, define also the same point in $\partial_{\mathscr{E}^{4}} \mathscr{M}^{4}$. It remains to show that there are no two different points $x, \bar{x}$ in $\partial_{c} \mathscr{M}^{4}$ which are identified in $\partial_{\mathscr{E}^{4}} \mathscr{M}^{4}$. From the way $\mathscr{M}^{4}$ is imbedded in $\mathscr{E}^{4}$, one can however see that this is impossible. (Remember that near $I^{0}, \mathscr{M}^{4}$ is the outside part of a light cone.)

\section{§ 4. Interpretation of Boundary Points}

In Section 2 it was shown how to attach a boundary to any conformal structure. How can we describe such boundaries in terms of the space-time manifold?

On the manifold $\mathscr{P}^{1}$, considered as a bundle over $\mathscr{P}$, a unique distribution of horizontal subspaces $H_{z}$ is defined. Using the parallelisation on $\mathscr{P}^{1}$ we can also define a unique horizontal subspace $\hat{H}_{z}$ if we consider $\mathscr{P}^{1}$ as a boundle over $\mathscr{M}$. The subspace $\hat{H}_{z}$ is defined as the subspace spanned by the fields $Z_{i}$, defined in $\S 2$.

With the help of $\hat{H}_{z}$ we can define lifts of vector fields and curves from $\mathscr{M}$ to $\mathscr{P}^{1}(\mathscr{M})$. There is however an essential difference between the distribution $\hat{H}_{z}$ and a connection in the principle bundle $\mathscr{P}^{1}(\mathscr{M})$. The distribution $\hat{H}_{z}^{z}$ is not invariant under the structure group, as it is shown in Appendix 2.

Therefore $\hat{H}_{z}$ defines no connection and - as we will see later - not any curve $x(\lambda), 0 \leqq \lambda \leqq 1$ has a horizontal lift $z(\lambda), 0 \leqq \lambda \leqq 1$. It may happen that a lift is only defined for $0 \leqq \lambda<\lambda_{0}<1$.

The following theorem shows that the points of $\partial_{c} \mathscr{M}$ are determined by horizontal curves in $\mathscr{P}^{1}(\mathscr{M})$.

Theorem. Let $z(\lambda)$ be an inextensible curve of finite length in $\mathscr{P}^{1}(\mathscr{M})$, $0 \leqq \lambda<1$. Then there exists a horizontal curve $w(\lambda), 0 \leqq \lambda<1$ of finite length contained in the fibres through $z(\lambda)$.

Proof. The proof is the complete analog of the corresponding statement for the $b$-boundary [1]. Therefore it will only be outlined:

Let $p:=\pi^{1} \circ \pi$ be the projection $\mathscr{P}^{1}(\mathscr{M}) \rightarrow \mathscr{M}$. The parallelisations on $\mathscr{P}^{1}(\mathscr{M})$ and the bundle over the Einstein universe $\mathscr{P}^{1}\left(\mathscr{E}^{4}\right)$ can be used to imbed $p^{-1}(p(z(\lambda)))$, i.e. the submanifold of all fibres through $z(\lambda)$, isometrically into $\mathscr{P}^{1}\left(\mathscr{E}^{4}\right)$. Because $\mathscr{P}^{1}\left(\mathscr{E}^{4}\right)$ is complete, the image of $z(\lambda)$ has an end point in $\mathscr{P}^{1}\left(\mathscr{E}^{4}\right)$ and a horizontal curve in $\mathscr{P}^{1}\left(\mathscr{E}^{4}\right)$ of finite length contained in the image of $p^{-1}(p(z(\lambda)))$ exists. Going back to $p^{-1}(p(z(\lambda)))$ one gets the horizontal curve of finite length because by the construction of the imbedding horizontal vectors are mapped onto horizontal ones. 
The theorem above shows that we have to understand the meaning of a horizontal curve in $\mathscr{P}^{1}(\mathscr{M})$ to get an interpretation for the points of $\partial_{c} \mathscr{M}$. Locally this is given by the following

Lemma. Let $z(\lambda)$ be a horizontal curve $0 \leqq \lambda \leqq 1$. Then there exists for any $\lambda_{0} \in[0,1]$ an $\varepsilon$ and a metric $g$ in the conformal class whose Ricci tensor vanishes on $p(z(\lambda)),-\varepsilon+\lambda_{0}<\lambda<\lambda_{0}+\varepsilon$, such that $z(\lambda)$ is given by $H_{u(\lambda)}$ where $H$ is the distribution of horizontal subspaces of the canonical connection of $g$, and $u(\lambda)=\pi^{1}(z(\lambda))$. The length of $z(\lambda),-\varepsilon+\lambda_{0}<\lambda<\lambda_{0}+\varepsilon$ is the generalised affine length [2] of $x(\lambda)$ calculated in the frame given by $u(\lambda)$.

Proof. Let $\varepsilon$ be determined by the condition that $x(\lambda)=p(z(\lambda))$ is a properly imbedded curve [6] for the $\varepsilon$-interval around $\lambda_{0}$. (This implies that there exists a coordinate system in which this part of $x(\lambda)$ is a coordinate line.)

If $g^{\prime}$ is an arbitrary metric in the conformal class then the condition that $g=e^{2 \sigma} g^{\prime}$ has vanishing Ricci tensor is that

$$
2 \sigma_{|i| \mid k}=-R_{i k}^{\prime}+2 \sigma_{\mid i} \sigma_{\mid k}-g_{i k}^{\prime}\left(\sigma_{\mid r} \sigma^{\mid r}-\frac{1}{6} R^{\prime}\right)
$$

holds along $x(\lambda)$. (Covariant derivatives and moving of indices with $g^{\prime}$.) Along $x(\lambda)$ this implies

$$
2 \sigma_{\mid i \| k} \dot{x}^{k}=-R_{i k}^{\prime} \dot{x}^{k}+2 \sigma_{\mid i} \sigma_{\mid k} \dot{x}^{k}-g_{i k}^{\prime} \dot{x}^{k}\left(\sigma_{\mid r} \sigma^{\mid r}-\frac{1}{6} R^{\prime}\right) .
$$

This is an ordinary 1 . order differential equation for $b_{k}(\lambda):=\sigma_{\mid k}(x(\lambda))$ along the curve $x(\lambda)$. Let $b_{k}(\lambda)$ be the solution with $b_{k}\left(\lambda_{0}\right)$ determined by the condition that $B_{i}-S_{i k}^{l}\left(b_{k}\left(\lambda_{0}\right)\right) E_{l}^{*}$ fixes $z\left(\lambda_{0}\right)$. A solution to these initial values exists in a certain interval around $\lambda_{0}$. Having determined $b_{k}(\lambda)$ we try to find a function $\sigma$ such that

$$
\sigma_{\mid k}(x(\lambda))=b_{k}(\lambda), \quad \sigma_{|k| l}(x(\lambda))=A_{k l}(\lambda)
$$

where $A_{k l}$ is determined by (4.1) with $\sigma_{\mid k}=b_{k}$. Because $b_{k}$ satisfies (4.2) and $x(\lambda)$ is properly imbedded one checks easily that $\sigma$ satisfying (4.3) exists (in fact one gets many solutions). Then by the very construction of $\sigma$ the metric $e^{2 \sigma} g^{\prime}$ has vanishing Ricci tensor along the piece of $x(\lambda)$ we are considering.

Let $f: \mathscr{P} \rightarrow \mathscr{P}^{1}$ be the section corresponding to the connection determined by $g=e^{2 \sigma} g^{\prime}$. Then this section is tangent to $H_{z}$ for any $z \in p^{-1}(x(\lambda))$ which is contained in the section. Let $u(\lambda), u\left(\lambda_{0}\right)=\pi^{1}\left(z\left(\lambda_{0}\right)\right)$ be the horizontal lift of $x(\lambda)$ into $\mathscr{P}(\mathscr{M})$ defined by the connection of $g$. Then the curve $\tilde{z}(\lambda)$ defined by $H_{u(\lambda)}, H_{u}$ being the horizontal subspace in $\mathscr{P}(\mathscr{M})$ of the connection of $g$, is clearly contained in the section $f$; hence 
$\dot{\tilde{z}}(\lambda) \in H_{z(\lambda)}$. Now $\pi_{*}^{1}(\dot{\tilde{z}}(\lambda))=\dot{u}(\lambda)$ and $\dot{u}(\lambda) \in H_{u(\lambda)}$ implies $\dot{\tilde{z}}(\lambda) \in \hat{H}_{z(\lambda)}$, hence $\tilde{z}(\lambda)$ is horizontal. By the construction of $\tilde{z}(\lambda), \tilde{z}\left(\lambda_{0}\right)=z\left(\lambda_{0}\right)$. Hence the uniqueness of horizontal lifts implies $z(\lambda)=\tilde{z}(\lambda)$. To verify the statement about the length of $z(\lambda)$ suppose

$$
\dot{z}(\lambda)=\alpha^{i}(\lambda) Z_{i},
$$

then by the definition of the metric on $\mathscr{P}^{1}$

Now

$$
\mathscr{L}=\int\left(\Sigma\left(\alpha^{i}\right)^{2}\right)^{\frac{1}{2}} d \lambda .
$$

$$
\pi_{*}^{1} \dot{z}=\dot{u}=\alpha^{i} B_{i}
$$

where $B_{i}$ are the standard horizontal fields of the connection of $g$ and this implies that $L$ is the length of $x(\lambda)$ calculated in a frame which is parallel in the metric $g$. This completes the proof.

If $z(\lambda)$ is a horizontal curve such that $p(z(\lambda))$ is properly imbedded then the theorem above implies that we can find a conformal factor $e^{2 \sigma}$ such that the Ricci tensor vanishes at all points of $x(\lambda)$. In general however this may not be possible. Take as an example a case in which $x(\lambda)$ is a closed null geodesic.

From the theorem, however, we learn that the conformal factor is only important on the curve, more precisely its gradient. Therefore, in general, one has to proceed in the following way to calculate a horizontal lift and its length:

Let $x(\lambda)$ be an arbitrary curve $0 \leqq \lambda \leqq 1$. Choose any metric $g$ in the conformal class and solve

$$
2 \nabla_{\dot{x}} b_{i}=-R_{i k} \dot{x}^{k}+2 b_{i} b_{k} \dot{x}^{k}-g_{i k} \dot{x}^{k}\left(b_{r} b^{r}-\frac{1}{6} R\right)
$$

for certain initial values $b_{k}(0)$. Then we get a 1 -form $b_{k}(\lambda)$ defined on $x(\lambda)$ for $0 \leqq \lambda<\lambda_{0}(\leqq 1)$. The non-linearity of $(4.5)$ is the reason that $b_{k}(\lambda)$ is not necessarily defined for $0 \leqq \lambda \leqq 1$. Once $b(\lambda)$ is determined, we propagate a frame $X_{s}^{i} \frac{\partial}{\partial x^{i}}$ along $x(\lambda)$ by the equation

$$
\frac{d X_{s}^{i}}{d \lambda}+\left(\Gamma_{k l}^{i}(x(\lambda))+S_{k l}^{i}\left(b_{r}(\lambda)\right)\right) X_{s}^{k} \dot{x}^{l}=0
$$

where $\Gamma_{k l}^{i}$ determines the connection of $g$. Then the frames $X_{s}^{i} \frac{\partial}{\partial x^{i}}$ and $b_{k}$ determine a horizontal lift. To get the length of the lift one has to calculate the length of $x(\lambda)$ in frame $X_{s}$.

Finally an example: Let us calculate some horizontal lifts of a time like geodesic in Minkowski space. Let $t(\lambda)=\lambda, 1 \leqq \lambda<\infty, x^{v}=0$ be the 
geodesic. If we now parallel propagate a frame with the flat connection we get a horizontal lift of infinite length.

We can however find a conformal factor $e^{2 \sigma}$ with $\sigma(t)$ such that $e^{2 \sigma} \eta$ has vanishing Ricci tensor along the geodesic. Equation (4.4) implies for $\sigma(t)$

$$
2 \ddot{\sigma}=2(\dot{\sigma})^{2}-(\dot{\sigma})^{2}
$$

which has the general solution, for $\dot{\sigma}(0) \neq 0$,

$$
\sigma=-2 \int_{1}^{t} \frac{d \lambda}{\lambda+c}+\sigma(1), \quad \dot{\sigma}(1)=\frac{-2}{1+c} .
$$

For $c>-1 \sigma(t)$ is defined for $1 \leqq \lambda<\infty$ and given by $\sigma=\ln (\lambda+c)^{-2}$. For the metric $e^{2 \sigma} \eta$ the curve $t(\lambda)=\lambda, x^{\nu}=0$ is still a geodesic whose length is

$$
\mathscr{L}=\int_{1}^{\infty} e^{\sigma(\lambda)} d \lambda=\int_{1}^{\infty}(\lambda+c)^{-2} d \lambda=\frac{1}{1+c}<\infty .
$$

Hence we have constructed lifts of finite length. We also realise that for $c \rightarrow-1$ the length of the lifts tends to infinity. One also derives easily from (4.6) that for $c<-1$ the conformal fact $\sigma$ is only defined for $1 \leqq \lambda<\lambda_{0}$ and that the corresponding lifts have infinite length.

\section{§5. Projective Boundary}

Two torsionfree linear connections $\Gamma, \Gamma^{\prime}$ are called projectively equivalent if any geodesic of $\Gamma$ can be parametrised such that it becomes a geodesic of $\Gamma^{\prime}$. An equivalence class $\mathscr{P}$ of projectively equivalent connection is a projective structure. If $\Gamma, \Gamma^{\prime} \in \mathscr{P}$, then in local coordinates it holds [9]

$$
\Gamma_{i k}^{\prime l}=\Gamma_{i k}^{l}+\delta_{i}^{l} b_{k}+\delta_{k}^{l} b_{i}
$$

where $b_{k} d x^{k}$ is a 1 -form. The relation (5.1) implies

$$
B_{i}^{\prime}=B_{i}-\left(\delta_{i}^{l} \tilde{b}_{k}+\delta_{k}^{l} \tilde{b}_{i}\right) E_{l}^{k}
$$

Comparing this with (2.4) in the conformal case, we realize that way in which the horizontal subspaces (in $\mathscr{L}(\mathscr{M})$ ) are related is quite similar. One proceeds by defining $\mathscr{P}^{1}$ as in the conformal case and gets again a horizontal subspace by the condition that the Ricci tensor vanishes. Hence there is again a parallelisation on $\mathscr{P}^{1}$ and we get a projective boundary of $\mathscr{M}^{4}$.

The sphere $\mathscr{S}^{4}$, with its natural projective structure, is the unique space admitting the maximal group of projective motions. The projective 
imbedding of Minkowski space into $\mathscr{S}^{4}$ shows that the projective boundary of Minkowski space is $\mathscr{S}^{3}$, hence the same as projective infinity defined by Eardley and Sachs $[4,5]$.

\section{Appendix 1}

Let $\mathscr{P}^{1}(\mathscr{P})$ be the bundle with fibre $\mathscr{R}^{4}$. A point $z \in \mathscr{P}^{1}(\mathscr{P})$ is a frame at $u=\pi_{1}(z)$ given by

$$
\left(B_{i}^{\prime}-S_{i l}^{k}(b) \stackrel{*}{E_{k}^{l}}\right)_{u}, \quad b \in \stackrel{\mathscr{*}^{4}}{ }{ }^{4} .
$$

The group $\mathscr{R}^{*}$ acts on $\mathscr{P}^{1}$ on the right: $u \rightarrow R_{c} u$, where $R_{c} u$ is the frame $\left(c \in \mathscr{R}^{4}\right)$

$$
\left(B_{i}^{\prime}-S_{i l}^{k}(b) \stackrel{*}{E}_{k}^{l}\right)_{u}-\left(S_{i l}^{k}(c) \stackrel{*}{E}_{k}^{l}\right)_{u}=\left(B_{i}^{\prime}-S_{i k}^{l}(b+c){ }^{*}{ }_{l}^{k}\right)_{u} .
$$

We defined an action of $\mathscr{C} \mathcal{O}$ on $\mathscr{P}^{1}$ in the following way. Any $a \in \mathscr{C} \mathcal{O}$ defines a map $R_{a}: \mathscr{P}(\mathscr{M}) \rightarrow \mathscr{P}(\mathscr{M})$. $\left(R_{a}\right)_{*}$ induces a map on the horizontal subspaces which maps $\mathscr{P}^{1}(\mathscr{P})$ into itself. Denote this map by $\hat{R}_{a}: \mathscr{P}^{1} \rightarrow \mathscr{P}^{1}$.

It will now be proved that

$$
R_{c^{\prime}} \circ \hat{R}_{a^{\prime}} \circ R_{c} \circ \hat{R}_{a}=R_{c^{\prime \prime}} \circ \hat{R}_{a^{\prime \prime}}
$$

holds. This implies that $\mathscr{R}^{4}$ and the action of $\mathscr{C} \mathcal{O}$ via $\hat{R}_{a}$ generate a Lie-transformation group $\mathscr{G}$ on $\mathscr{P}^{1}$.

Clearly this action of the group is free, and the orbits are $\left(\pi \circ \pi^{1}\right)^{-1}(x)$, $x \in \mathscr{M}$. The local triviality of $\mathscr{P}^{1}(\mathscr{P})$ and $\mathscr{P}(\mathscr{M})$ implies that $\mathscr{P}^{1}$ is a principle bundle over $\mathscr{M}$ with group $\mathscr{G}$. If we consider $\mathscr{P}^{1}$ as a bundle over $\mathscr{M}$ we denote it by $\mathscr{P}^{1}(\mathscr{M})$.

Let us now prove (A 1.3).

Take any $z_{0} \in \mathscr{P}^{1}$ and choose a connection $\Gamma$ such that $H_{u_{0}}$ for $u_{0}=\pi_{1}\left(z_{0}\right)$ defines $z_{0}$. Then $H_{u_{0}}$ is spanned by $\left(B_{i}\right)_{u_{0}}$, where $B_{i}$ are the standard horizontal fields of $\Gamma$. By the definition of $R_{a}$, we get that

$$
\hat{R}_{a}\left(z_{0}\right)=\left(R_{a}\right)_{*} H_{z_{0}}=H_{u_{0} a} .
$$

The subspace $H_{u_{0} a}$ is spanned by $\left(B_{i}\right)_{u_{0} a}$. From the definition of $R_{c}$ acting on $\mathscr{P}^{1}$ one finds

$$
\left(R_{c} \circ \hat{R}_{a}\right)\left(z_{0}\right)=\left(B_{i}\right)_{u_{0} a}-S_{i k}^{l}\left(c_{r}\right)\left(\ddot{E}_{l}^{k}\right)_{u_{0} a} .
$$

To find the subspace defining $\left(\hat{R}_{a^{\prime}} \circ R_{c} \circ \hat{R}_{a}\right)\left(z_{0}\right)$ we apply $\left(R_{a^{\prime}}\right)_{*}$ and use the transformation properties

$$
\left(R_{a}\right)_{*} B_{i}=a_{i}^{\prime-1 k} B_{k}, \quad\left(R_{a^{\prime}}\right)_{*} \stackrel{*}{k}_{l}^{k}=a_{u}^{\prime k}{ }^{*}{ }_{v}^{u} a_{l}^{\prime-1 v} .
$$

Hence we obtain

$$
\left(\hat{R}_{a^{\prime}} \circ R_{c} \circ \hat{R}_{a}\right)\left(z_{0}\right)=a_{i}^{\prime-1 t}\left(B_{t}\right)_{u_{0} a a^{\prime}}-S_{i k}^{l}\left(c_{r}\right) a_{u}^{\prime k}{ }_{E_{v}^{u}}^{*} a_{l}^{\prime-1 v} .
$$


Finally we apply $R_{c^{\prime}}$. To do this we have to take into account that the $i$-th vector in (A 1.7) does not project onto the $i$-th vector of the frame $u_{0} a a^{\prime}$. This is however true for the vectors

$$
\left(B_{t}\right)_{u_{0} a a^{\prime}}-a_{t}^{\prime i} S_{i k}^{l}\left(c_{r}\right) a_{u}^{\prime k} E_{v}^{u} a_{l}^{\prime-1 v} .
$$

Applying $R_{c^{\prime}}$ we now get

$$
\begin{gathered}
\left(R_{c^{\prime}} \circ \hat{R}_{a^{\prime}} \circ R_{c} \circ \hat{R}_{a}\right)\left(z_{0}\right)=\left(B_{t}\right)_{u_{0} a a^{\prime}} \\
-a_{t}^{i} a_{u}^{\prime k} S_{i k}^{l}(c) a_{l}^{\prime-1 v} E_{v}^{u} \\
-S_{t u}^{v}\left(c^{\prime}\right) E_{v}^{u} .
\end{gathered}
$$

Using $S_{i k}^{l}(c)=\delta_{i}^{l} c_{k}+\delta_{k}^{l} c_{i}-\eta_{i k} \eta^{l s} c_{s}$ and the fact that $a_{i}^{k} \in \mathscr{C} \mathcal{O}$, one finds by a short calculation that (A 1.9) can be written as

$$
\left(R_{c^{\prime}} \circ \hat{R}_{a^{\prime}} \circ R_{c} \circ \hat{R}_{a}\right)\left(z_{0}\right)=\left(B_{t}\right)_{u_{0} a a^{\prime}}-S_{t u}^{v}\left(a_{r}^{\prime j} c_{j}+c_{r}^{\prime}\right) \stackrel{E}{*}_{v}^{u} .
$$

Comparing this expression with (A 1.5) we get

$$
\left(R_{c^{\prime}} \circ \hat{R}_{a^{\prime}} \circ R_{c} \circ \hat{R}_{a}\right)\left(z_{0}\right)=\left(R_{c^{\prime \prime}} \circ \hat{R}_{a^{\prime \prime}}\right)\left(z_{0}\right)
$$

where

$$
a^{\prime \prime}=a a^{\prime}, \quad c_{r}^{\prime \prime}=a_{r}^{\prime j} c_{j}+c_{r}^{\prime} .
$$

As $a^{\prime \prime}, c^{\prime \prime}$ are independent of $z_{0}$, (A 1.11) holds for any $z_{0}$, and (A 1.11) implies (A 1.3). We realise furthermore that $\mathscr{G}$ is a semi-direct product of $\mathscr{C} \mathcal{O}$ and $\mathscr{R}^{4}$, where $\mathscr{C} \mathcal{O}$ acts as a group of automorphisms on $\mathscr{R}^{4}$.

\section{Appendix 2}

In Section 2 a parallelisation $Z_{i}, Z_{\alpha}, \stackrel{A}{*}^{m}$ was defined on $\mathscr{P}^{1}$. In this appendix it will be shown how these fields transform under the action of the group $\mathscr{G}$ defined in Appendix 1.

Lemma 1. Let $\stackrel{*}{\alpha}_{\alpha}$ be the basis of $\mathscr{\mathscr { C }} \mathcal{O}$ defined in $\S 2,(2.9)$. Let $M_{\alpha}$ be the corresponding vector field on $P^{1}$, defined by the action of $\mathscr{C} \mathcal{O}$ on $\mathscr{P}^{1}$. Then it holds $Z_{\alpha}=\stackrel{*}{\alpha}_{\alpha}$.

Proof. Let $z_{0}$ be any point in $\mathscr{P}^{1}(\mathscr{P})$. Then there exists $g \in \mathscr{C}$ such that: 1) the Ricci tensor vanishes at $\left(\pi \circ \pi^{1}\right)\left(z_{0}\right)=: x_{0}$, and 2) the crosssection $f: \mathscr{P} \rightarrow \mathscr{P}^{1}$ defined by the connection of $g$ passes through $z_{0}$. This implies that the section $f$ is tangent to the horizontal subspace $H_{z}$ for all points projecting onto $x^{0}$ under $\pi \circ \pi^{1}$.

The element $M_{\alpha} \in \mathscr{C} \mathscr{O}$ generates a 1 -parameter subgroup $a(t)$ of $\mathscr{C} \mathcal{O}$. By the very definition of $M_{\alpha}^{*}, E_{\alpha}^{*}$ it follows that $E_{\alpha}^{*}\left(u_{0}\right), u_{0}=\pi^{1}\left(z_{0}\right)$ is tangent of the curve $R_{a(t)} u_{0}$ at $t=0$ and $M_{\alpha}\left(z_{0}\right)$ is tangent to $\hat{R}_{a(t)} z_{0}$ at $t=0 . \hat{R}_{a(t)} z_{0}$ is contained in the Section $\mathrm{f}$, is horizontal and projects 
onto $R_{a(t)} u_{0}$. This implies $Z_{\alpha}=\stackrel{M}{\alpha}_{\alpha}$, using the definition of $Z_{\alpha}$. Knowing that $\stackrel{*}{A}^{m}, Z_{\alpha}=\stackrel{*}{*}_{\alpha}$ are the fundamental vector field tangent to the fibres in $\mathscr{P}^{1}(\mathscr{M})$ one has the following transformation property [8]:

If $g$ is any element of $\mathscr{G}$, and $R_{g}$ the corresponding transformation on $\mathscr{P}^{1}(\mathscr{M})$, then

$$
\begin{aligned}
& \left(R_{g}\right)_{*} \stackrel{*}{A^{m}}=\left(\operatorname{ad}\left(g^{-1}\right) A^{m}\right)^{*}, \\
& \left(R_{g}\right)_{*} \stackrel{*}{M_{\alpha}}=\left(\operatorname{ad}\left(g^{-1}\right) M_{\alpha}\right)^{*},
\end{aligned}
$$

where ad is the adjoint representation of $\mathscr{G}$.

The transformation properties of $Z_{i}$ are given by

Lemma 2. For $a=\left(a_{k}^{i}\right) \in \mathscr{C} \mathcal{O}, c=\left(c_{r}\right) \in \mathscr{R}^{4}$ we have

$$
\begin{aligned}
& \left(\hat{R}_{a}\right)_{*} Z_{i}=\left(a^{-1}\right)_{i}^{k} Z_{k}, \\
& \left(R_{c}\right)_{*} Z_{i}=Z_{i}+\beta_{i}^{\alpha}(c) Z_{\alpha}+\left(-c_{i} c_{m}+\frac{1}{2} \eta_{i m} c_{s} c^{s}\right) A^{*} .
\end{aligned}
$$

Proof. Choose a point $z_{0} \in \mathscr{P}^{1}$ and construct a section $f$ as in the beginning of the proof of Lemma 1 . Then $\left.\left(\hat{R}_{a}\right)_{*} Z_{i}\right|_{z_{0}} \in T_{z_{0} a}\left(P^{1}\right)$ is again contained in the horizontal subspace $H_{z_{0} a}$.

Suppose

$$
\left.\left(\hat{R}_{a}\right)_{*} Z_{i}\right|_{z_{0}}=\left(\alpha_{i}^{k} Z_{k}+\beta_{i}^{\alpha} Z_{\alpha}\right)_{z_{0} a} .
$$

Projecting onto $\mathscr{P}$ we find

$$
B_{i}=\alpha_{i}^{k} a_{k}^{j} B_{j}+\beta_{i}^{\alpha}{\stackrel{*}{E_{\alpha}},}
$$

and this implies $\alpha_{i}^{k}=\left(a^{-1}\right)_{i}^{k}, \beta_{i}^{\alpha}=0$.

The vector $\left(R_{c}\right)_{*}\left(Z_{i}\right)_{z_{0}}$ is not horizontal because it is contained in the section $R_{c} \circ f$ in $\mathscr{P}^{1}$, which is not defined by a metric connection. Suppose

$$
\left(R_{c}\right)_{*}\left(Z_{i}\right)_{z_{0}}=\alpha_{i}^{k} Z_{k}+\beta_{i}^{\alpha} Z_{\alpha}+\gamma_{i m} \stackrel{*}{A^{m}} .
$$

Projecting onto $\mathscr{P}$ we get

$$
B_{i}=\alpha_{i}^{k}\left(B_{k}-S_{k j}^{l}\left(c_{r}\right) \stackrel{*}{E}_{l}^{j}\right)+\beta_{i}^{\alpha} \stackrel{*}{E}_{\alpha}
$$

Hence

$$
\alpha_{i}^{k}=\delta_{i}^{k}, \quad S_{i j}^{l}\left(c_{r}\right) \stackrel{*}{E}_{l}^{j}=\beta_{i}^{\alpha} E_{\alpha}^{*} .
$$

Clearly $\beta_{i}^{\alpha}$ depends only on $c_{r}$ and not on $z_{0}$.

It remains to show that $\gamma_{i m}$ in (A 2.5) also depends only on $c_{r}$.

Let us first calculate a cross-section $h$ which passes through $H_{z_{0} c}$. Such a section is given by the connection of a metric $e^{2 \sigma} g$ such that the Ricci tensor of $e^{2 \sigma} g$ vanishes at $x_{0}$ and $\tilde{\sigma}_{\mid k}\left(u_{0}\right)=c_{r}$ holds.

Then $\left(Z_{i}+\beta_{i}^{\alpha} Z_{\alpha}\right)_{z_{0} c}$ is tangent to $h$ and $\left(R_{c}\right)_{*}\left(Z_{i}\right)_{z_{0}}$ is tangent to $R_{c} \circ f$. The two sections $R_{c} \circ f, h$ define a map $\varphi: \mathscr{P} \rightarrow \mathscr{R}^{4}$ by

$$
\left(R_{c} \circ f\right)(u)=R_{\varphi(u)} \circ h(u) .
$$


If $u(t)$ is a curve in $\mathscr{P},\left(R_{c} \circ f\right)(u(t)), h(u(t))$ the image curve in the sections, then the tangent vectors are related by

$$
\left(\left(R_{c} \circ f\right)(u(o))\right)^{\cdot}=h(u(o))^{\cdot}+\varphi_{m}(u(o))^{\cdot} \stackrel{*}{A^{m}} .
$$

In the case we are considering $\varphi(u)$ is given by $u \rightarrow-\tilde{\sigma}_{\mid r}(u)+c_{r}$.

Hence the tangent of the curve in $\mathscr{R}^{4}$ is

Using

$$
\left.\frac{d}{d t}\left(-\tilde{\sigma}_{\mid r}(u(t))\right)\right|_{t=0} \stackrel{*}{*}^{r}
$$

$$
\frac{d}{d t}\left(-\tilde{\sigma}_{\mid r}(u(t))\right)_{t=0}=\dot{u}(o) \tilde{\sigma}_{\mid r}
$$

where $\tilde{\sigma}_{\mid r}$ is considered as function on $\mathscr{P}$, one finds easily, taking $u(t)$ to be tangent to $B_{i}$

$$
\gamma_{i m}=\left(-B_{i} \tilde{\sigma}_{\mid r}\right)_{u_{0}}=\left(-\widetilde{\sigma_{|r| \mid}}\right)_{u_{0}} .
$$

The conformal factor $\sigma$ was determined by the condition $\tilde{\sigma}_{\mid r}\left(u_{0}\right)=c_{r}$ and the vanishing of the Ricci tensor of $e^{2 \sigma} \mathrm{g}$. From this we find

$$
\left(\widetilde{\sigma_{\mid r \| i}}\right)_{u_{0}}=c_{r} c_{i}-\frac{1}{2} \eta_{r i} c_{s} c^{s} \text {. }
$$

Therefore we get finally

$$
\gamma_{i n}=-c_{i} c_{m}+\frac{1}{2} \eta_{i m} c_{s} c^{s}
$$

which completes the proof of the lemma.

We found that, applying $R_{c}, \hat{R}_{a}$ to the parallelisation, the new fields are linear combinations of the old with coefficient constant on $\mathscr{P}^{1}$, where the coefficients depend on $c, a$. This implies as it was shown in [1] that the map $R_{g}$ is uniformly continuous with respect to the distance defined by the metric in which the parallelisation is orthonormal. This is the property we used in Section 2.

\section{Appendix 3}

In [14], Kuiper shows that the Einstein universe admits a 15parameter group of global conformal motions. A more geometrical derivation of this result is given here.

Consider $\mathscr{R}^{n}$ as a vector space with a non degenerate scalar product

$$
g=\sum_{\alpha=1}^{p}\left(x^{\alpha}\right)^{2}-\sum_{A=1}^{q}\left(t^{A}\right)^{2} .
$$

Let $\mathcal{O}(p, q)$ be the orthogonal group which keeps $g$ invariant. Let $\mathscr{N}$ be the submanifold of $\mathscr{R}^{n}$ defined by

$$
\mathscr{N}:=\left\{\left(x^{\alpha}, t^{A}\right) \mid \Sigma\left(x^{\alpha}\right)^{2}-\Sigma\left(t^{A}\right)^{2}=0,\left(x^{\alpha}, t^{A}\right) \neq 0\right\} .
$$

The manifold $\mathcal{N}$ is the analog of the light cone of a Lorentz metric. 
Define an equivalence relation on $\mathscr{R}^{n}$ by $x^{i} \sim \bar{x}^{i}$ if $x^{i}=\lambda \bar{x}^{i}, \lambda>0$. Then $\mathscr{R}^{n} / \sim$, i.e. the space of directions, is diffeomorphic to $\mathscr{S}^{n-1}$. $\left(\mathscr{R}^{n} / \sim\right.$ is a double covering of the projective space $\mathscr{P}^{n-1}$.)

Let $\mathscr{Q}$ be the image of $\mathscr{N}$ in $\mathscr{R}^{n} / \sim$ under the projection $\pi: \mathscr{R}^{n} \rightarrow \mathscr{R}^{n} / \sim$. Since $\mathcal{O}(p, q)$ maps isotropic lines in $\mathscr{N}$ into isotropic lines we get an action of $\mathcal{O}(p, q)$ on $\mathscr{2}$ by

$$
\tilde{A}:=\pi \circ A \circ \pi^{-1}, \quad A \in \mathcal{O}(p, q) .
$$

Clearly $A \rightarrow \tilde{A}$ is an isomorphism ${ }^{2}$.

The metric $g$ on $\mathscr{R}^{n}$ induces a degenerate metric on $\mathscr{N}$. Let $\mathscr{H}$ be the group of linear transformation $x^{i} \rightarrow \lambda x^{i}, \lambda>0$. Then $\mathscr{H}$ consists of conformal transformations of $g$, whose restrictions to $\mathscr{N}$ are diffeomorphisms with $\pi \circ h=\pi$.

It will now be shown that the degenerate metric induced by $g$ on $\mathscr{N}$ defines a conformal structure on $\mathscr{Q}$ of signature $(p-1, q-1)$.

Let $X$ be a tangent vector at a point $\alpha \in \mathscr{Q}$ and $\tilde{X}_{u}, \tilde{Y}_{v}$ two tangent vectors of $\mathscr{N}$ at $u, v \in \pi^{-1}(\alpha)$ projecting onto $X$. What is the relation between $g\left(\tilde{X}_{u}, \tilde{X}_{u}\right), g\left(\tilde{Y}_{v}, \tilde{Y}_{v}\right)$ ?

As $\tilde{X}_{u}, \tilde{Y}_{v}$ project on the same vector under $\pi$, there is $h \in \mathscr{H}$ such that

$$
h_{*} \tilde{X}_{u}=\tilde{Y}_{v}+L
$$

where $L$ is tangent to the null generator of $\mathscr{N}$ through $v$. Recalling that $h$ is a conformal transformation for $g$ we find

$$
\lambda^{2} g\left(\tilde{X}_{u}, \tilde{X}_{u}\right)=g\left(\tilde{Y}_{v}, \tilde{Y}_{v}\right) .
$$

Hence a unique conformal structure is induced on $\mathscr{2}$, and this conformal structure is invariant under the action of $\mathcal{O}(p, q)$ given by $\tilde{A}$, because $\tilde{A}$ is an isometry of $g$.

Let us describe a metric in the conformal structure on $\mathscr{Q}$ explicitely: If we define $r^{2}=\Sigma\left(x^{\alpha}\right)^{2}, \varrho^{2}:=\Sigma\left(t^{A}\right)^{2}$, the metric $g$ can be written on a part of $\mathscr{R}^{n}$ as

$$
g=d r^{2}+r^{2} d \Sigma^{2}(p-1)-d \varrho^{2}-\varrho^{2} d \Sigma^{2}(q-1) \quad \begin{aligned}
& \varrho>0 \\
& r>0
\end{aligned}
$$

where $d \Sigma^{2}(m)$ denotes the metric of an unit $m$-sphere $\mathscr{S}^{m} . \mathscr{N}$ is the submanifold $\varrho^{2}=r^{2}>0$ and the degenerate metric induced on $\mathscr{N}$ is

$$
r^{2}\left(d \Sigma^{2}(p-1)-d \Sigma^{2}(q-1)\right) .
$$

$(\varrho, r$ are always positive on $\mathscr{N})$. The points $r=$ const define a section through $\mathcal{N}$, hence the conformal structure on $\mathscr{Q}$, which has the topology

\footnotetext{
${ }^{2}$ Provided $p \geqq 1, q \geqq 1$ and $p+q \geqq 3$.
} 
$\mathscr{S}^{p-1} \times \mathscr{S}^{q-1}$, can be represented by the metric $d \Sigma^{2}(p-1)-d \Sigma^{2}(q-1)$. It is therefore conformally flat.

Finally let us consider the case $p=4, q=2$. Then $\mathscr{Q}=\mathscr{S}^{3} \times \mathscr{S}^{1}$ and the conformal structure is given by the Lorentz metric

$$
d \Sigma^{3}(3)-d \Sigma^{2}(1)
$$

which admits $\mathcal{O}(4,2)$ as a global group of conformal transformations. If we pass to the universal covering space of $\mathscr{S}^{3} \times \mathscr{S}^{1}$ together with the induced metric and the induced transformations we get the Einstein universe on which a covering group of $\mathcal{O}(4,2)$ acts.

Remark. If one uses $\mathscr{P}^{n-1}$ instead of $\mathscr{R}^{n} / \sim$ one also gets a conformal structure invariant under $\mathcal{O}(p, q)$. In the case $p=4, q=2$, this is compactified Minkowski space which also has the topology $\mathscr{S}^{3} \times \mathscr{S}^{1}$, and the space $\mathscr{2}$ considered above is a double covering of compactified Minkowski space.

Acknowledgement. I would like to thank Jürgen Ehlers and Martin Walker for their most helpful discussions and careful reading of the manuscript.

\section{References}

1. Schmidt, B.G.: G.R.G. 1, 269-280 (1971)

2. Hawking, S. W., Ellis, G.F.R.: The large scale structure of the Universe. Cambridge: University Press 1973

3. Penrose,R.: Structure of space-time, in Battelle Rencontres, ed. by C.M.DeWitt, J.A.Wheeler, 121-235. New York: Benjamin 1969

4. Eardley,D., Sachs, R. K.: J. Math. Phys. 14, 2 (1973)

5. Eardley,D.: Ph. D. Thesis, University of California. Berkeley 1971

6. Sternberg,S.: Lectures on differential geometry. Englewood Cliffs, New Jersey: Prentice-Hall, Inc. 1964

7. Kobayashi,S.: Transformation groups in differential geometry. Berlin-HeidelbergNew York: Springer 1972

8. Kobayashi,S., Nomizu, K.: Foundations of differential geometry, Vol. I. New York: Interscience 1969

9. Eisenhart,L.P.: Riemannian geometry. Princeton: Princeton University Press 1925

10. Schmidt,B.G.: Differential geometry, in relativity, Astrophysics and Cosmology, ed. by W.Israel. Dortrecht-Holland: D. Reidel Publishing Company, 1974

11. Jordan, P., Ehlers, J., Kundt, W.: Akad. Wiss. Mainz, Abh. Math.-Nat. Kl. Nr. 2 (1960)

12. Hocking, J. G., Young, G. S.: Topology. London: Addison-Wesley 1961

13. Penrose, R.: Proc. Roy. Soc. (London) A 284 (1965)

14. Kuiper, N.H.: Ann. Math. 50, 916 (1949)

15. Geroch,R., Kronheimer,E.H., Penrose, R.: Proc. Roy. Soc. (London) A 327 (1972)

Communicated by J. Ehlers

B. G. Schmidt

MPI für Physik und Astrophysik

D-8000 München 40

Föhringer Ring 6

Federal Republic of Germany 